INFLAMMATORY BOWEL DISEASE

\title{
Pancolonic indigo carmine dye spraying for the detection of dysplasia in ulcerative colitis
}

\author{
M D Rutter, B P Saunders, G Schofield, A Forbes, A B Price, I C Talbot
}

Gut 2004;53:256-260

See end of article for authors' affiliations

.....................

Correspondence to: Dr B Saunders, Wolfson Unit for Endoscopy, St Mark's Hospital, Watford Rd, Harrow, Middlesex HA1 3UJ, UK;

b.saunders@ic.ac.uk

Accepted for publication 9 July 2003
Background and aims: Colonoscopic surveillance for cancer in longstanding extensive ulcerative colitis relies heavily on non-targeted mucosal biopsies. Chromoendoscopy can aid detection of subtle mucosal abnormalities. We hypothesised that routine pancolonic indigo carmine dye spraying would improve the macroscopic detection of dysplasia and reduce the dependence on non-targeted biopsies.

Patients and methods: One hundred patients with longstanding extensive ulcerative colitis attending for colonoscopic surveillance underwent "back to back" colonoscopies. During the first examination, visible abnormalities were biopsied, and quadrantic non-targeted biopsies were taken every $10 \mathrm{~cm}$. Pancolonic indigo carmine $(0.1 \%)$ was used during the second colonoscopic examination, and any additional visible abnormalities were biopsied.

Results: Median extubation times for the first and second colonoscopies were 11 and 10 minutes, respectively. The non-targeted biopsy protocol detected no dysplasia in 2904 biopsies. Forty three mucosal abnormalities (20 patients) were detected during the pre-dye spray colonoscopy of which two (two patients) were dysplastic: both were considered to be dysplasia associated lesions/masses. A total of 114 additional abnormalities (55 patients) were detected following dye spraying, of which seven (five patients) were dysplastic: all were considered to be adenomas. There was a strong trend towards statistically increased dysplasia detection following dye spraying ( $p=0.06$, paired exact test). The targeted biopsy protocol detected dysplasia in significantly more patients than the non-targeted protocol $(p=0.02$, paired exact test).

Conclusions: No dysplasia was detected in 2904 non-targeted biopsies. In comparison, a targeted biopsy protocol with pancolonic chromoendoscopy required fewer biopsies (157) yet detected nine dysplastic lesions, seven of which were only visible after indigo carmine application. Careful mucosal examination aided by pancolonic chromoendoscopy and targeted biopsies of suspicious lesions may be a more effective surveillance methodology than taking multiple non-targeted biopsies.
1 n 1925, Crohn and Rosenberg first observed that ulcerative colitis could be complicated by colorectal carcinoma. ${ }^{1}$ Subsequent studies have confirmed the increased rate of colorectal cancer, particularly in patients with extensive ulcerative colitis of at least 10 years' duration. To address this risk, many centres perform colonoscopic surveillance of such patients. Surveillance protocols recommend multiple non-targeted "random" biopsies throughout the colon to detect dysplasia, a precursor of cancer, based on initial evidence that dysplasia could occur in flat mucosa and often occurred as a widespread "field effect" distant to cancer sites in $96-100 \%$ of cases. $^{23}$ The American Society for Gastrointestinal Endoscopy guidelines advise taking two to four non-targeted biopsies for every $10 \mathrm{~cm}$ of colon and rectum. ${ }^{4}$ However, this is time consuming, expensive, and often logistically difficult for hard pressed colonoscopists and histopathologists.

More recent studies have cast doubt on the precept of widespread dysplasia, with several studies reporting that approximately one quarter of cases of colorectal carcinoma in ulcerative colitis occur without detectable dysplasia distant to the tumour. ${ }^{5-8}$ It is still widely believed that dysplasia is invisible at endoscopy, ${ }^{9}$ but anecdotally we have also formed the impression that most dysplasia detected during surveillance has come from colonoscopically visible abnormalities, although lesions may be sessile, flat, or minimally elevated, and require meticulous examination to detect them.

Endoscopic dye spraying of the gastrointestinal tract (chromoendoscopy) with a contrast dye such as indigo carmine can highlight subtle architectural irregularities in colonic mucosa, improving the sensitivity of the endoscopic examination. This may be particularly important in colonoscopic surveillance in ulcerative colitis where the early changes of dysplasia can be subtle. Preliminary work has indicated that high resolution videoendoscopy with indigo carmine dye spray may increase the yield of detected flat neoplastic lesions in ulcerative colitis. ${ }^{10}$

We hypothesised that the routine use of pancolonic indigo carmine dye spray in colonoscopic surveillance of ulcerative colitis would improve the macroscopic detection of dysplasia, improving the diagnostic yield of the procedure while reducing the dependence on non-targeted biopsies.

The aims of this study were therefore:

(1) to assess the efficacy of pancolonic indigo carmine dye spray in increasing the yield of dysplasia in patients with longstanding ulcerative colitis undergoing colonoscopic surveillance; and

(2) to determine the proportion of dysplastic lesions detected from targeted biopsies of macroscopically visible abnormalities, as opposed to non-targeted biopsies of background colonic mucosa.

In this study, pancolonic dye spraying was utilised to aid the detection of mucosal irregularities, as opposed to the categorisation of such lesions once found. As it is critical for

Abbreviations: DALM, dysplasia associated lesion/mass 
any surveillance examination to minimise the number of false negative procedures (that is, to minimise the chance that dysplasia is overlooked), the study was designed to maximise the sensitivity of the colonoscopic surveillance examination, and not to maximise the specificity of biopsy specimens for dysplasia. Thus all mucosal irregularities were biopsied, and not just those areas which were felt to be dysplastic.

\section{MATERIALS AND METHODS Patients}

The study size was calculated at 100 patients, based on a predye spray dysplasia detection of $8 \%$ of patients and an assumption that dye spraying would detect all lesions seen on the pre-dye spray colonoscopy, and would double the rate of detection $(90 \%$ power, alpha $=0.05)$. Ethics approval was obtained from the local research ethics committee. All patients attending St Mark's Hospital between January and August 2002 for routine colonoscopic surveillance of ulcerative colitis were invited to participate. The colonoscopic surveillance programme includes all patients with macroscopic colitis extending proximal to the splenic flexure of at least eight years' duration. Consenting patients were interviewed, and patient demographics recorded.

\section{Colonoscopy}

Each patient underwent back to back colonoscopic examinations, the first constituting the current "gold standard" colonoscopic surveillance examination, and the second using pancolonic indigo carmine dye spray. All colonoscopies were performed by a single experienced endoscopist (MDR) using Olympus 240 series Innoflex (variable stiffness) colonoscopes.

Standard doses of intravenous sedation (median doses pethidine $25 \mathrm{mg}$ and midazolam $1.25 \mathrm{mg}$ ) and antispasmodic medication (median dose buscopan $20 \mathrm{mg}$ ) were given at the discretion of the endoscopist at the beginning of the procedure. Further doses of intravenous medication were given as clinically required during the procedure. Insertions and withdrawals were timed. Faecal fluid residue was aspirated on the first insertion to ensure optimal mucosal views. The quality of bowel preparation was documented as good (only liquid stool present, removable by suction), fair (some semi-formed stool obscuring less than 5\% of the mucosa after suction), or poor (solid stool obscuring over $5 \%$ of the mucosa after suction).

Once the caecal pole had been reached on initial intubation, meticulous inspection of the entire colonic mucosa was performed on withdrawal, without the use of dye spray. To minimise the possibility of missed lesions, a very careful extubation examination technique was employed, incorporating multiple positional shifts of the patient and extra intravenous antispasmodic medication as required. At $10 \mathrm{~cm}$ intervals throughout the colon, the mucosa was photographed, and quadrantic non-targeted colonic biopsies taken, in accordance with the American Society for Gastrointestinal Endoscopy guidelines. ${ }^{4}$ In addition, any suspicious area of mucosa was photographed and biopsied or removed, as clinically indicated. Suspicious areas were defined as any mucosal irregularity that was not felt to be entirely consistent with chronic or active ulcerative colitis, regardless of whether or not it was felt to be dysplastic. In patients with more than 10 macroscopically unequivocal post-inflammatory polyps, representative biopsies were taken, along with biopsies from any atypical polyps.

When the tip of the colonoscope had been withdrawn to the anal margin, the colonoscope was reinserted to the caecal pole. On the second extubation, the lumen was sprayed in a segmental fashion using $0.1 \%$ indigo carmine delivered via a specially designed dye spray catheter (Olympus PW-5V1). After allowing a few seconds for the dye to settle onto the mucosal surface, excess pools of indigo carmine were suctioned. The mucosa was then scrutinised, and any abnormalities not identified on the initial examination were biopsied or removed, as clinically indicated, and documented as before.

Thus three series of biopsies were taken, categorised as: (1) "non-targeted quadrantic"; (2) "pre-dye spray targeted"; and (3) "dye spray targeted". All biopsy specimens were analysed by one of two experienced gastrointestinal histopathologists, who were blinded to the protocol used to obtain the biopsy. Any specimen showing dysplasia was independently reported by both histopathologists, and in the event of interobserver variation a consensus opinion was reached.

\section{RESULTS}

A total of 108 consecutive patients were invited to participate in the study, of whom 101 consented. In one case the study was abandoned prior to the second intubation at the patient's request, owing to a technically difficult initial total colonoscopy. Thus 100 patients completed the study.

\section{Patient demographics}

There were 61 male and 39 female patients. Median age of patients was 53 years (range 33-79); median age at onset of ulcerative colitis was 27 years (range 7-67); and the median duration of colitis was 24 years (range 8-52). In 11 patients, the study examination was their index screening procedure whereas 89 patients had undergone previous colonoscopic surveillance procedures. The documented proximal extent of macroscopic inflammation was the transverse colon in 12 patients, hepatic flexure in four patients, ascending colon in one patient, and pancolonic in 83 patients.

\section{Colonoscopic details}

The quality of bowel preparation was good in 82 patients, fair in 15, and poor in three patients. Median colonic length after shortening and straightening the colonoscope at the caecal pole was $70 \mathrm{~cm}$ (range 50-100). Median time for initial intubation was eight minutes (range 2-38) and three minutes for the second intubation (range 1-21). Median extubation times were 11 minutes (range 4-18) and 10 minutes (range 4-22) for the initial and second procedures, respectively (no significant difference, $\mathrm{p}=0.13$; paired $t$ test). Mean volume of indigo carmine used was $72 \mathrm{ml}$ (range 35100). The histological description of colitis was well healed (neither chronic nor active inflammation) in 32 patients, quiescent (chronic inflammation only) in 39 patients, and showing active inflammation (neutrophil leucocytes infiltrating cryptal or surface epithelium) in 29 patients. Active inflammation was graded as mild in 21 cases, moderate in seven, and severe in one case. No complications were reported.

Non-targeted quadrantic biopsies

A total of 2904 non-targeted biopsies were taken, a mean of 29 per patient. No dysplasia was detected in any of these biopsies.

\section{Targeted biopsies}

Overall, 157 suspicious mucosal areas were detected in 61 patients. Forty three abnormalities (from 20 patients) were detected during the pre-dye spray colonoscopy, and following indigo carmine dye spraying 114 additional abnormalities (in 55 patients) were detected. Median size was $4 \mathrm{~mm}$ (range 1-40). Six of the abnormalities were pedunculated, 69 were sessile, 75 were flat topped elevated abnormalities, and seven abnormalities were described as irregular appearing mucosa. 
Pre-dye spray targeted biopsies

Of the 43 abnormalities detected during the pre-dye spray colonoscopy, nine lesions were hyperplastic polyps and 32 were inflammatory or post-inflammatory polyps. Two patients had dysplastic lesions: a $20 \mathrm{~mm}$ sessile lesion on quiescent mucosa at the hepatic flexure in a 71 year old male with no previous dysplasia and a $15 \mathrm{~mm}$ sessile lesion on mildly inflamed mucosa in the sigmoid colon in an 80 year old female with previous dysplasia, who has repeatedly declined surgery unless cancer was detected. Targeted biopsies showed low grade dysplasia, confirming the endoscopist's impression that these were dysplasia associated lesions/masses (DALMs) (fig 1).

Dye spray targeted biopsies

Both DALM lesions were visible after indigo carmine dye spraying. Of the 114 additional abnormalities detected following dye spraying, seven were dysplastic (from five patients). Five of these were tubular adenomas with low grade dysplasia (fig 2), and two were serrated adenomas with low grade dysplasia. Three of the lesions were described as flat lesions and four were sessile. The size of these well circumscribed adenomas ranged from 2 to $6 \mathrm{~mm}$. Two adenomas were found in the caecum, two at the hepatic flexure, two in the transverse colon, and one in the descending colon. Two of the adenomas occurred proximal to the extent of colitis and five were within the UC extent (four in well healed disease, one in an area of mild inflammation). Of the other 107 abnormalities detected following dye spraying, 41 were hyperplastic polyps, 65 post-inflammatory and inflammatory polyps, and one was described as villiform mucosa but without dysplasia.

\section{Dysplasia detection summary}

With regard to dysplasia detection, the non-targeted biopsy protocol (2904 biopsies) detected no dysplasia from 100 patients, the pre-dye spray targeted biopsy protocol (43 biopsies) detected two dysplastic lesions in two of the 100 patients, and the dye spray targeted biopsy protocol (114 biopsies) detected these two dysplastic lesions plus seven additional dysplastic lesions in five more of the 100 patients. Thus overall, dysplasia was detected in $7 \%$ of patients. There was a strong statistical trend towards an increase in dysplasia detection with dye spraying (7/100 patients $v 2 / 100$ patients; $\mathrm{p}=0.06$, paired exact test). Compared with the non-targeted biopsy protocol, the targeted biopsies detected dysplasia in

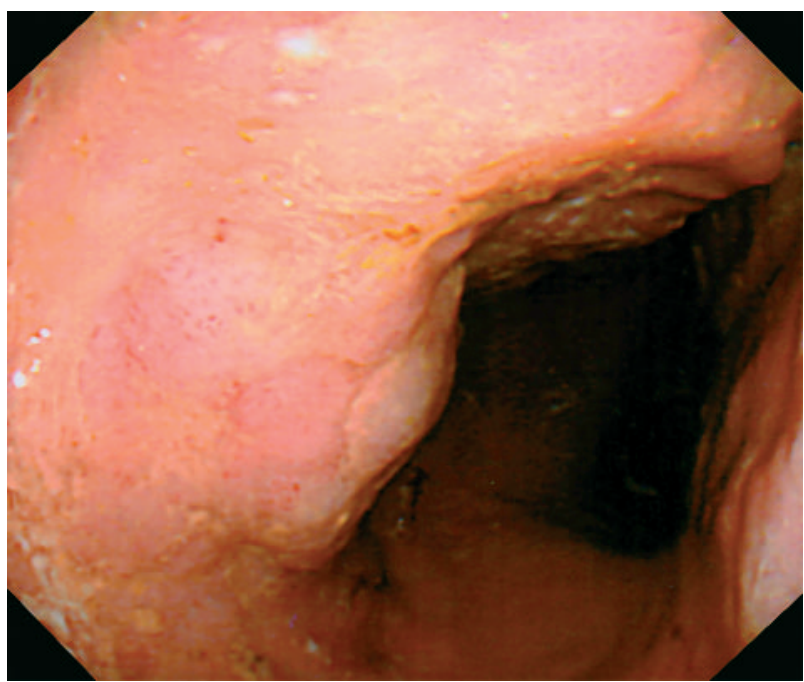

Figure 1 Dysplasia associated lesion/mass.

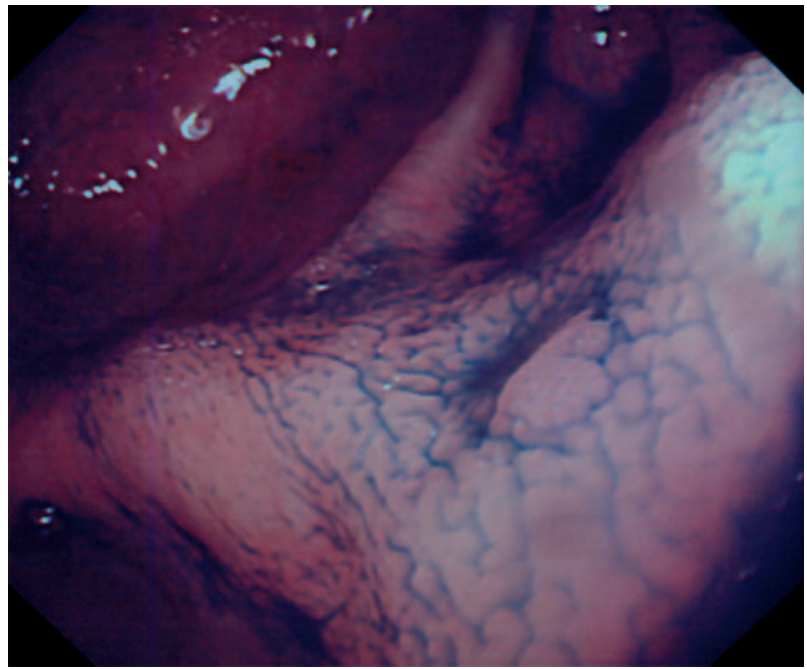

Figure 2 Dysplastic lesion highlighted by indigo carmine dye spray.

significantly more patients (7/100 patients $v$ 0/100 patients; $\mathrm{p}=0.02$, paired exact test $)$.

\section{DISCUSSION}

Since the early 1970s, colonoscopic surveillance of ulcerative colitis has relied heavily on the detection of premalignant dysplastic tissue by non-targeted biopsies. Rubin et al calculated that 33 biopsies were required from a single procedure to detect dysplasia with $90 \%$ confidence. ${ }^{11}$ In practice, this number of specimens is seldom achieved. ${ }^{12} \mathrm{~A}$ recent survey of British gastroenterologists revealed 57\% took 10 or fewer biopsies per colonoscopy, and only $2 \%$ took more than $20,{ }^{13}$ perhaps reflecting fiscal and time constraints both at colonoscopy and in the pathology department, and perceived lack of benefit. In this study, we adhered to the American Society for Gastrointestinal Endoscopy guidelines which suggest quadrantic non-targeted biopsies for every $10 \mathrm{~cm}$ of colon, equating to $20-40$ biopsies per colon. ${ }^{4}$

Despite taking over 2900 non-targeted biopsies in 100 patients during the study, no episode of dysplasia was detected from a non-targeted biopsy. These findings echo those of a retrospective study examining the value of nontargeted biopsies in the detection of gastric neoplasia, which concluded that routine biopsies not targeted at a visible lesion did not increase the detection of gastric malignancy. ${ }^{14}$

Endoscopic dye spraying of the gastrointestinal tract (chromoendoscopy) was first described by Tada in $1976 .{ }^{15}$ Indigo carmine is a contrast dye which neither reacts with nor is absorbed by the mucosa, but simply pools in the mucosal grooves and pits allowing better topographic definition. In conjunction with improved colonoscopic equipment, this has the potential to improve the diagnostic yield by detecting inconspicuous lesions that might otherwise have been overlooked. This might be particularly important in surveillance in ulcerative colitis where the early changes of dysplasia can be subtle. Our study showed that the routine use of indigo carmine dye spray throughout the whole colon is simple, feasible, and safe.

We used back to back colonoscopies for our study. With this approach each patient acts as their own control, giving the most accurate assessment of the additional benefit of dye spraying over conventional colonoscopy. To our knowledge, this is the first time such an approach has been used. It is possible that some of the lesions seen on the dye spray colonoscopy could have been seen on the first (non-dye spray) colonoscopy but were not detected ("missed lesions"). 
In studies of back to back colonoscopies in non-colitic populations, the miss rate for adenomas has been reported as $15-24 \% .{ }^{16}$ We tried to minimise the possibility of missed lesions by adhering to a meticulous examination technique, as already described. We are confident that dysplastic lesions seen on the second examination were not missed lesions, as this would have meant a miss rate of $350 \%$. One of the benefits of a dye spray examination is that it is much clearer to see which areas have not been examined, and therefore when used as the sole examination, for this reason alone it may reduce the number of missed lesions.

Chromoendoscopy significantly increased detection of suspicious mucosal areas. In our study, an additional 114 abnormalities were detected by the technique, although the vast majority of these (107/114) were not dysplastic and therefore probably of no clinical significance. In this study we made no attempt to macroscopically categorise individual lesions as dysplastic or not-the aim of our study was to increase the detection of subtle lesions. Chromoendoscopy did however detect seven additional dysplastic polyps in five patients. These well circumscribed lesions were considered endoscopically and histologically to be adenomas. Where such lesions occur proximal to the extent of the colitis, it is clear they can be treated as sporadic adenomas and removed endoscopically with no long term increase in cancer. ${ }^{17}$ When visible dysplastic lesions occur within the area of colitis, treatment is more controversial. The initial description of a DALM referred to any visible dysplastic lesion within an area of inflammation, excluding "typical tubular adenomas $1 \mathrm{~cm}$ or less", and reported subsequent colorectal cancer in seven of the 12 patients with such lesions. ${ }^{18}$ Other studies, including from our own centre, corroborated the high risk of cancer in patients with such lesions and suggested that colectomy was indicated in this scenario. ${ }^{19}$ More recently however, there has been growing evidence that a proportion of such lesions can safely be removed endoscopically without excess cancer risk. ${ }^{17}{ }^{20}$ We were confident that the small well circumscribed lesions detected after dye spraying in our study were endoscopically resectable. What is less clear is what would have happened to these lesions had they been left in situ. The natural history of such lesions within an area of colitic mucosa is poorly understood: it might be that they follow no more aggressive a course than sporadic adenomas, but it is possible that their course is more aggressive, indeed they may be precursors of larger DALMs. Either way, these lesions have malignant potential and dye spraying permitted their early detection and removal.

Overall, the incidence of dysplasia might be considered low in this study. However, it is similar to that of the St Mark's surveillance programme as a whole ( 7 of 100 colonoscopies versus 209 of 2627 colonoscopies (unpublished data); $\mathrm{p}=0.87$ Fisher's exact test, two sided). In an apparently similar cohort of patients, Jaramillo et al detected a much higher rate of dysplasia (23 in 85 patients) despite using indigo carmine in a focused rather than pancolonic manner. ${ }^{10}$ It is unlikely that the difference in dysplasia detection is due to variation in examination technique as the overall detection of polyps was lower than in our study ( 104 abnormalities in 85 patients compared with 157 in 100 patients). Jaramillo et al also reported a high detection rate of dysplasia from nontargeted biopsies (22\% of patients), suggesting either different patient characteristics in terms of cancer risk or different histopathological criteria for the diagnosis of dysplasia.

One of the perceived drawbacks of chromoendoscopy is that it prolongs the procedure. However in our study, a dye spray extubation with targeted biopsies took the same time as a standard extubation with multiple non-targeted biopsies. The targeted biopsy protocol required far fewer biopsies (157, of which nine were dysplastic, compared with 2904, of which none was dysplastic). Thus in terms of dysplasia yield, the colonoscopist's time might be better spent on careful scrutiny of the mucosa with dye spray, rather than taking 20-40 nontargeted biopsies. New techniques such as optical biopsying using laser fluorescence spectroscopy, which allows tissue characterisation without the need for physical biopsying, might further reduce the need for multiple colonic biopsies. $^{21} 22$

Although we feel it unwise to advocate complete abolition of non-targeted biopsying, our study demonstrates the poor dysplasia yield in unselected surveillance patients, and we suggest that particular attention should be paid to careful colonoscopic inspection with targeted biopsies of all abnormalities, rather than relying on non-targeted biopsies. Clearly, as with all colonoscopic surveillance examinations, a meticulous endoscopic examination is essential to ensure adequate mucosal views throughout and to minimise the chance of missing lesions. Dye spraying may be of additional benefit in this respect.

In summary, we have shown that indigo carmine dye spraying of the whole colon is feasible and safe, and in terms of dysplasia detection may be more time effective than taking large numbers of non-targeted biopsies. Colonoscopists should not rely on non-targeted biopsies detecting dysplasia but should focus more on careful examination of the mucosa, which can be aided by pancolonic indigo carmine dye spray.

\section{Authors' affiliations}

M D Rutter, B P Saunders, G Schofield, A Forbes, Wolfson Unit for Endoscopy and Department of Gastroenterology, St Mark's Hospital, Harrow, UK

A B Price, I C Talbot, Department of Gastrointestinal Histopathology, St Mark's Hospital, Harrow, UK

\section{REFERENCES}

1 Crohn BB, Rosenberg $\mathrm{H}$. The sigmoidoscopy picture of chronic ulcerative colitis (non-specific). Am J Med Sci 1925;170:220-8.

2 Morson BC, Pang LS. Rectal biopsy as an aid to cancer control in ulcerative colitis. Gut 1967;8:423-34.

3 Hulten L, Kewenter J, Ahren C. Precancer and carcinoma in chronic ulcerative colitis. A histopathological and clinical investigation. Scand J Gastroenterol 1972; 7:663-9.

4 The role of colonoscopy in the management of patients with inflammatory bowel disease. American Society for Gastrointestinal Endoscopy. Gastrointest Endosc 1998:48:689-90.

5 Connell WR, Talbot IC, Harpaz N, et al. Clinicopathological characteristics of colorectal carcinoma complicating ulcerative colitis. Gut 1994;35:1419-23.

6 Taylor BA, Pemberton JH, Carpenter HA, et al. Dysplasia in chronic ulcerative colitis: implications for colonoscopic surveillance. Dis Colon Rectum 1992:35:950-6.

7 Woolrich AJ, DaSilva MD, Korelitz BI. Surveillance in the routine management of ulcerative colitis: the predictive value of low-grade dysplasia. Gastroenterology 1992;103:431-8.

8 Ransohoff DF, Riddell RH, Levin B. Ulcerative colitis and colonic cancer. Problems in assessing the diagnostic usefulness of mucosal dysplasia. Dis Colon Rectum 1985;28:383-8.

9 Sharan R, Schoen RE. Cancer in inflammatory bowel disease. An evidencebased analysis and guide for physicians and patients. Gastroenterol Clin North Am 2002:31:237-54.

10 Jaramillo E, Watanabe M, Befrits R, et al. Small, flat colorectal neoplasias in long-standing ulcerative colitis detected by high-resolution electronic video endoscopy. Gastrointest Endosc 1996:44:15-22.

11 Rubin CE, Haggitt RC, Burmer GC, et al. DNA aneuploidy in colonic biopsies predicts future development of dysplasia in ulcerative colitis. Gastroenterology 1992;103:1611-20.

12 Connell WR, Lennard-Jones JE, Williams CB, et al. Factors affecting the outcome of endoscopic surveillance for cancer in ulcerative colitis. Gastroenterology 1994;107:934-44.

13 Eaden JA, Ward BA, Mayberry JF. How gastroenterologists screen for colonic cancer in ulcerative colitis: an analysis of performance. Gastrointest Endos 2000;51:123-8.

14 Cadman B, Dixon MF, Wyatt Jl. Value of routine, non-targeted biopsies in the diagnosis of gastric neoplasia. J Clin Pathol 1997;50:832-4.

15 Tada M, Katoh S, Kohli Y, et al. On the dye spraying method in colonofiberoscopy. Endoscopy 1976;8:70-4.

16 Rex DK, Cutler CS, Lemmel GT, et al. Colonoscopic miss rates of adenomas determined by back-to-back colonoscopies. Gastroenterology 1997; 112:24-8. 
17 Rubin PH, Friedman S, Harpaz N, et al. Colonoscopic polypectomy in chronic colitis: conservative management after endoscopic resection of dysplastic polyps. Gastroenterology 1999;117:1295-300.

18 Blackstone MO, Riddell RH, Rogers BH, et al. Dysplasia-associated lesion or mass (DALM) detected by colonoscopy in long-standing ulcerative colitis: an indication for colectomy. Gastroenterology 1981;80:366-74.

19 Bernstein CN, Shanahan F, Weinstein WM. Are we telling patients the truth about surveillance colonoscopy in ulcerative colitis? Lancet 1994;343:71-4.
20 Engelsgierd M, Farraye FA, Odze RD. Polypectomy may be adequate treatment for adenoma-like dysplastic lesions in chronic ulcerative colitis Gastroenterology 1999;117:1288-94.

21 Kapadia CR, Cutruzzola FW, O'Brien KM, et al. Laser-induced fluorescence spectroscopy of human colonic mucosa. Detection of adenomatous transformation. Gastroenterology 1990;99:150-7.

22 Romer TJ, Fitzmaurice M, Cothren RM, et al. Laser-induced fluorescence microscopy of normal colon and dysplasia in colonic adenomas: implications for spectroscopic diagnosis. Am J Gastroenterol 1995;90:81-7.

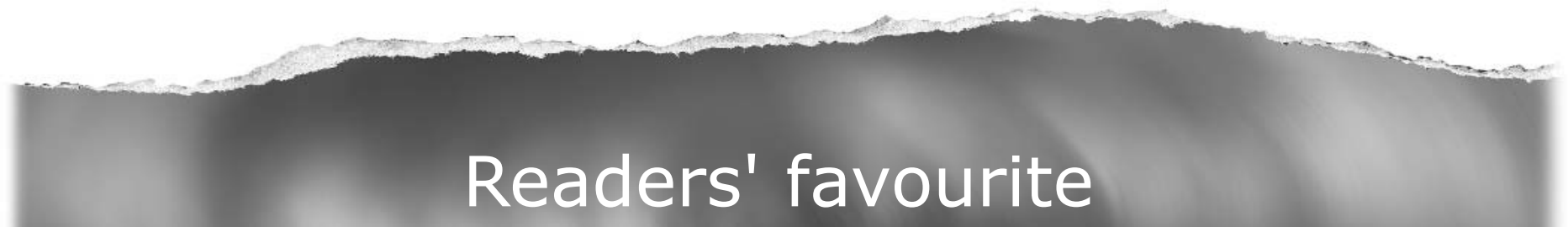

Top 10

Click on the "Top 10" button on the homepage to see which are the best read articles each month

www.gutjnl.com 